\title{
Molecular Identification of Pathogen Yeast from Star Gooseberry (Phyllanthus acidus) and Cucumber (Cucumis sativus L.) Extracts
}

\section{Ridawati, Alsuhendra, and Yati Setiati}

Faculty of Engineering, Universitas Negeri Jakarta, Jalan Rawamangun Muka, Kampus A, Gd. H. Lt.2. 13220

\section{Abstract}

In this study, two yeast strains, isolated from spontaneously fermented extracts of cucumber and star gooseberry fruits, were characterized by molecular methods. The identification of yeast isolates at the species level performed with sequence-based analysis of the region spanning the $D 1$ and $D 2$ regions (D1/D2) of the large ribosomal subunit (LSU) allowed for more accurate identification of yeast species. The yeasts identification proceeds based on the sequencing of the polymerase chain reaction (PCR)-amplified 600 base pair D1/D2 region of the yeast 26S ribosomal DNA, which

Corresponding Author:

Ridawati

ridawati.sesil@gmail.com

Received: 11 January 2019

Accepted: 14 February 2019

Published: 25 March 2019

Publishing services provided by Knowledge E

(c) Ridawati et al. This article is distributed under the terms of the Creative Commons

Attribution License, which permits unrestricted use and redistribution provided that the original author and source are credited.

Selection and Peer-review under the responsibility of the 3rd ICTVET 2018 Conference Committee.

\section{G OPEN ACCESS} was compared by a BLAST search to the D1/D2 regions of all validly described yeast species on file in the GenBank database, facilitating accurate and rapid identification. This research identified the yeasts from spontaneous fermentation of extract star gooseberry and cucumber fruits as Candida tropicalis (100\%, 637 bp) and Kodama ohmeri (99\%, 539bp), respectively. Both of the yeasts were pathogen yeast in fruits and humans.

Keywords: molecular identification, star gooseberry, cucumber

\section{Introduction}

Phyllanthus acidus, commonly known as star gooseberry, is a widely distributed plant in Indonesia and other Asian countries. Fruit drupaceous, oblate, 1-1.5 cm x (2-2.5) cm when fresh. At the branch tips are clusters of deciduous, greenish or pinkish branchlets, thin, green and smooth on the upper surface, blue-green with a bloom on the underside; shallowly 6- or 8-lobed, greenish yellow to creamy-white; flesh firm, sour with a hard, bony, grooved stone containing 6-8 smooth seeds. Star gooseberry (Phyllanthus acidus) fruit is rich in antioxidants including ascorbic acid and phenolic compounds. The mature sour fruits may be eaten fresh, candied in sugar, pickled, fruit juice and flavor dishes. The fruit juice is used in cold drinks and fruit to make vinegar. Extraction is a key operation in juice processing. 
Another Indonesian fruit that is usually served as appetizers or desserts is a cucumber. Cucumber (Cucumis sativus L) fruit is composed mostly of water; more than $96 \%$ of the edible unpeeled fruit is water. Other constituents of Cucumber are vitamins, minerals, amino acids, phytosterols, phenolic acids, fatty acids, and cucurbitacin. Glycosides, steroids, flavonoids, carbohydrates, terpenoids, and tannins identified in an aqueous extract of the cucumber fruit. They associated with cooling, healing, soothing and emollient effects.

Yeast is widely dispersed in nature with a wide variety of habitats. They are commonly found on plant leaves, flowers, and fruits, as well as a sugary product. Yeasts are also found as parasites and infectious. Besides infections, yeast is very useful in commercial application. The research about isolation, identification and characterization yeast from the fruit of various countries has been reported [1-3]. Molds and yeasts tolerate high-osmotic and low-pH conditions and grow at refrigeration temperatures and can, therefore, cause spoilage in the processed product. Typical yeast species found in citrus juices are Candida parapsilosis, Candida stellata, Saccharomyces cerevisiae, Torulaspora delbrueckii, and Zygosaccharomyces rouxii, although species from the genus Rhodotorula, Pichia, Hanseniaspora, and Metschnikowia are also common [4]. The reason that will be finding out novel yeast from fruit was to produce bread which can bring on unprecedented taste and flavor, and further to bring it production. Many types of yeasts are used for making many foods, baker's yeast in bread production; brewer's yeast in beer fermentation; and yeast in wine fermentation.

The yeast identification has been performed using biochemical analysis, substrate assimilation methods, morphological examination, or various combinations of the three. Conventional methods of yeast identification are often time-consuming and difficult [5]. Recently, the various molecular identification methods for yeasts by the use of rRNA genes, with the ITS regions and D1/D2 regions has been reported [6].

This research aimed to identify and characterize the isolate from star gooseberry spontaneous fermented and cucumber spontaneous fermented by molecular identification methods.

\section{Materials and Methods}

\subsection{Sample preparation}

Star gooseberry fruits were randomly selected from the local trees in Jakarta, and cucumber fruits were purchased locally. All chemical reagents used in this study were 
purchased from Merck (Germany). The fruits were washed and crushed in a blender. The obtained fruit mash was mixed with water at the weight ratio of $1 / 2$, adjusted to $\mathrm{pH} 3.0$ and added $15 \%$ sucrose. The solution was incubated in a water bath at $37^{\circ} \mathrm{C}$ for three days.

\subsection{Isolation of yeast}

After three days, yeast isolation was carried out using Yeast Malt Agar (YMA) medium containing ( $\mathrm{g} / \mathrm{L}$ ); $3 \mathrm{~g}$ of yeast extract, $3 \mathrm{~g}$ of malt extract, $5 \mathrm{~g}$ of peptone, $10 \mathrm{~g}$ of glucose and $15 \mathrm{~g}$ of agar. Yeast isolation method using spread plate and dilution technique with two repetitions. One $\mathrm{ml}$ of sample from each dilution was spread on YMA media to isolate yeast. The growth of yeast was observed. Check isolated colonies microscopically to identify yeast. Look especially for non-fuzzy opaque pasty-looking white or pink colonies. Pick a well-isolated colony and restream onto a fresh plate. Pick a well-isolated colony and make detailed drawings of different-shaped cells seen microscopically.

\subsection{Molecular identification of yeast}

The preparation of yeast DNA templates for Polymerase Chain Reaction (PCR) reactions was following the last methods by modification [7]. For PCR reaction with PCR Master Mix (Promega).

The universal primer used to amplify the D1/D2 region of the LSU rRNA gene for forwarding is the NL1 (5'-GCATATCAATAAGCGGAGGAAAAG-3') and NL4 (5'-GGTCCGT GTT TCAAGACGG-3').

For extraction of DNA, one loopful of cells was suspended in $300 \mu \mathrm{L}$ MilliQ water in microtubes and were homogenized using vortex (Bio-Rad: BR 2000, California, USA). The DNA was extracted by using the boiling method of [8].

PCR reaction was carried out under the following conditions: 1 cycle at $95{ }^{\circ} \mathrm{C}$ for 4 minutes; 35 cycles at $94^{\circ} \mathrm{C}$ for 1 minute; $53^{\circ} \mathrm{C}$ for 45 seconds, and $72{ }^{\circ} \mathrm{C}$ for 1 minute 15 seconds; 1 cycle at $72{ }^{\circ} \mathrm{C}$ for 8 minutes. Cycle Sequencing reaction using the Thermo Sequenase Primary Cycle

Sequencing (Pharmacia) kit and the Big DyeTerminator v3.1 kit Cycle Sequencing Ready Reaction (Applied Biosystems). 
The Cycle Sequencing reaction is carried out in the following conditions: 20 cycles at $95^{\circ} \mathrm{C}$ for 30 seconds; $55^{\circ} \mathrm{C}$ for 30 seconds, and $72^{\circ} \mathrm{C}$ for 1 minute. Sequence data collection is carried out with A.L.F. Express Pharmacia automated DNA sequencer.

Cycle sequencing reaction using Big Dye Terminator v3.1 Cycle Sequencing Ready Reaction kit (Applied Biosystems) performed in the following conditions: 25 cycles at $96^{\circ} \mathrm{C}$ for 10 seconds; $50^{\circ} \mathrm{C}$ for 5 seconds, and $60^{\circ} \mathrm{C}$ for 1.5 minutes.

Sequence data collection is carried out with the automated DNA sequencer $A B I$ Prism 3100 Genetic Analyzer (Applied Biosystems). Regional sequence data of the D1 / D2 LSU rRNA gene obtained were sent via the internet to seek homology with the same regional sequence of microorganisms stored in the DNA database (DDBJ, EMBL or GenBank) through the BLAST program to find out their identity.

The CLUSTAL Program W is used to sort sequence data. Distance matrix of sequence data that has been aligned (aligned data) is calculated using the two method parameters of Kimura. Phylogenetic tree construction using the neighbor-joining (NJ) method. The strength of each phylogenetic tree branch is estimated by bootstrapping 1,000 times replication.

\section{Results}

In this research, yeast isolates were obtained from spontaneously fermented extracts of cucumber and star gooseberry fruits. The growth of yeast was shown in Figure 1. The shape especially for non-fuzzy opaque pasty-looking white or cream colonies.

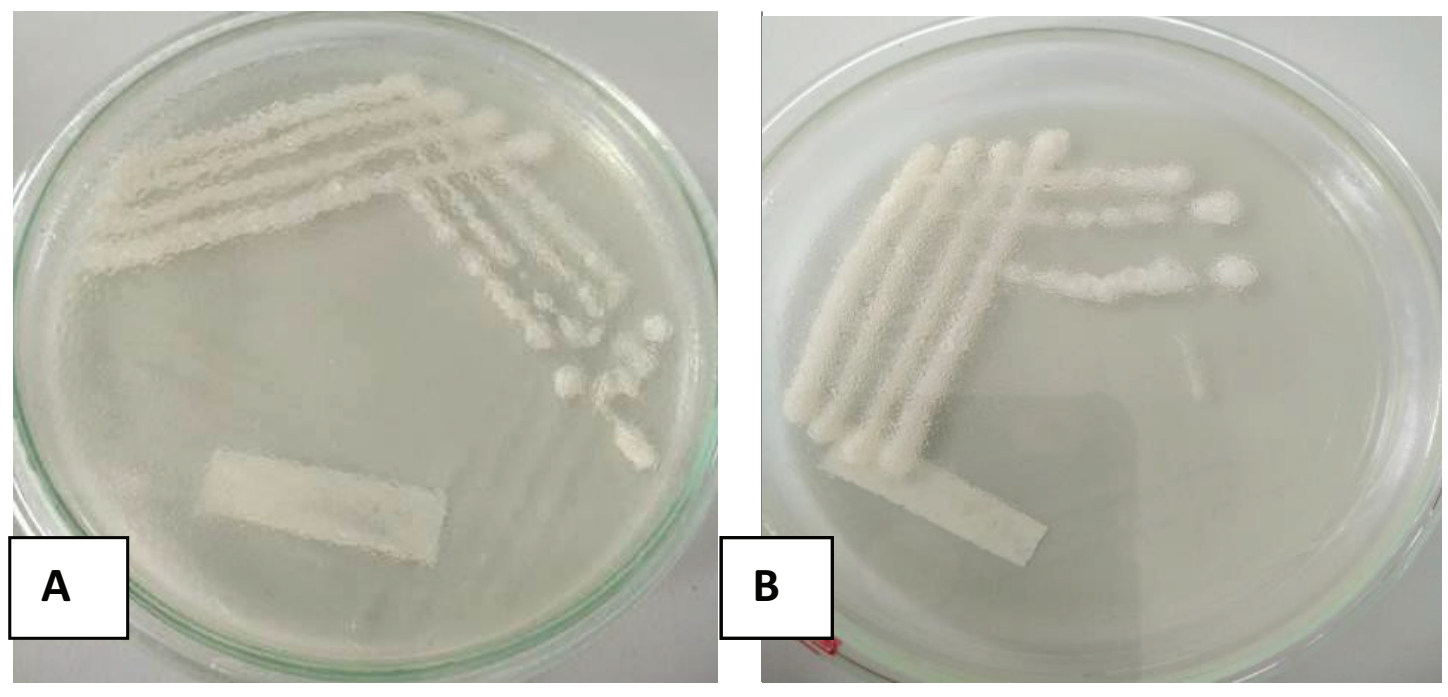

Figure 1: The growth of yeast isolates in YMA (24 hours, $\left.37^{\circ} \mathrm{C}\right) ;(\mathrm{A})$ isolate $\mathrm{C}$ from the star gooseberry spontaneously fermented extract and (B) Isolate $\mathrm{T}$ from the cucumber spontaneously fermented extract. 
This study was viewing the specimen under high magnification (1000x). The oval (egg- shaped) organism, which was the yeast, shown in Figure 2.

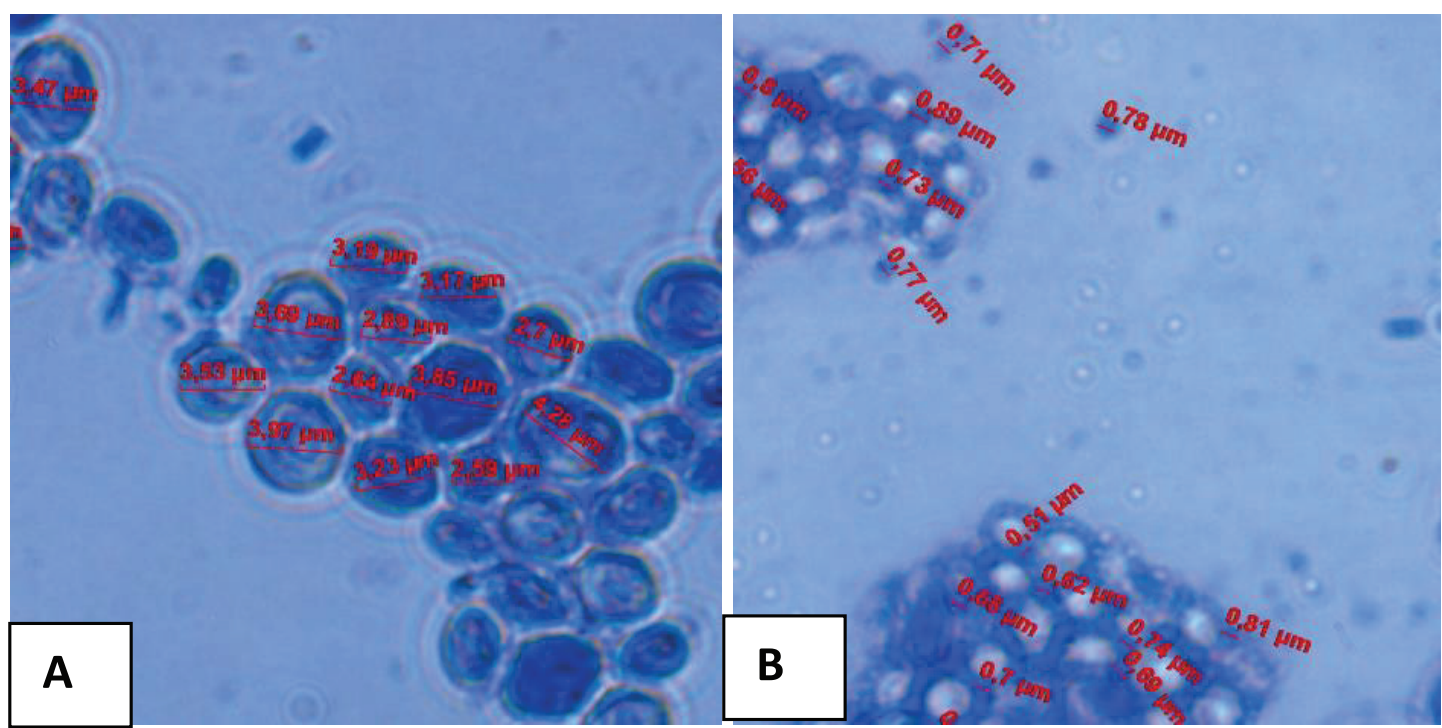

Figure 2: Morphology cell from isolate $C(A)$ and isolate $T(B)$.

The relationship between yeast isolates from Candida tropicalis, Kodama ohmeri and their closely related species are shown in the phylogenetic tree using the NJ method (Figure 3.).

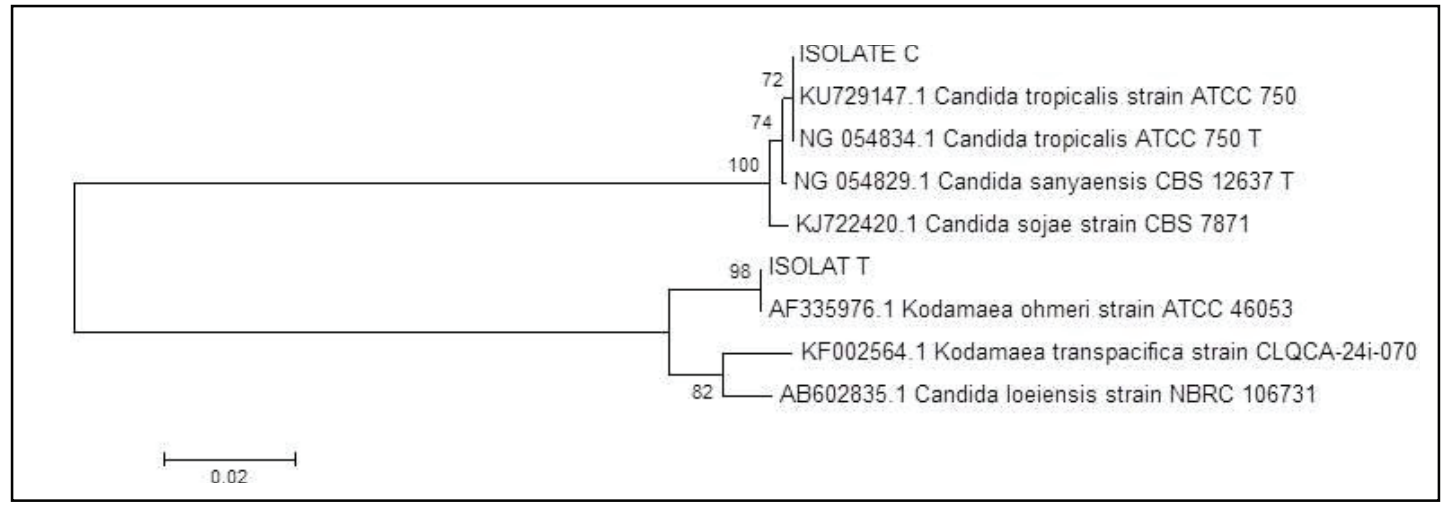

Figure 3: Neighbor-joining of phylogram of the various polymorphic sequences of the D1/D2 domains of the large-subunit rDNA of Kodama ohmeri and Candida tropicalis. Bootstrap values greater than $50 \%$ from 1,000 replicate bootstrap resampling.

\section{Discussion}

In this research, yeast isolates were obtained from spontaneously fermented extracts of cucumber and star gooseberry fruits. The shape especially for non-fuzzy opaque pasty-looking white or cream colonies. This study was viewing the specimen under high magnification (1000x). The oval (egg-shaped) organism, which was the yeast.. The yeast 
from star gooseberry and cucumber shown in Figure 2, were with diameters of between $2,64-4,28 \mu \mathrm{m}$ and $0,56-0,81$, respectively. The both of yeast had variation and smaller size than Saccharomyces cerevisiae. Temperature-induced variability of cellular size, volume, intracellular granularity, a fraction of budding cells of yeast Saccharomyces cerevisiae [9]. The lengths and shapes are approached in different ways in different fields; they serve as a read-out for classifying genes or proteins in cell biology whereas they result from scaling arguments in condensed matter physics [10].

The yeast isolates were identified based on D1/D2 regions of rDNA. The isolates were identified as Kodama ohmeri and Candida tropicalis. Based on sequence data of D1/D2 regions of rDNA, the yeast isolates from a star gooseberry spontaneously fermented extract of Candida tropicalis showed a high degree of similarity (100\%) to their closest species. The yeast isolates from a cucumber spontaneously fermented extract of Kodama ohmeri showed a high degree of similarity (99\%). The relationship between yeast isolates from Candida tropicalis, Kodama ohmeri and their closely related species are shown in the phylogenetic tree using the $\mathrm{NJ}$ method (Figure 3.). Our NJ tree showed that the yeast isolates are phylogenetically diverse and distributed in the phyla of Ascomycota.

In this research, the phylogenetic tree constructed from rDNA-D1/D2 sequencing showed that the yeasts from classes Candida and Kodama were found. Isolate $C$ from star gooseberry was identically 100\% with Candida tropicalis strain ATCC 750 and Candida tropicalis strain ATCC 750 T. Its closed to Candida sanyasis CBS 12637 (99\%) and Candida sojae strain CBS 7871. Isolate T from cucumber was identically $100 \%$ with Kodama ohmeri strain ATCC 46053. Its closed to Kodama transpacific strain CLQCA 24 i070 and Candida loeiensis strain NBRC 106731. Kodama ohmeri was a pathogen yeast that isolated from a patient in the hospital [11].

\section{Conclusion}

This research identified the pathogen yeasts from spontaneous fermented of extract star gooseberry and cucumber as Candida tropicalis (100\%, 637 bp) and Kodama ohmeri (99\%, 539bp), respectively.

\section{Funding}

This work was supported by the Ministry of Research, Technology and Higher Education, Indonesia for the research grant under Grant No.12/SP2H/DRPM/LPPMUNJ/II/2018. 


\section{Acknowledgment}

The authors would like to thank their colleagues for their contribution and support to the research. They are also thankful to all the reviewers who gave their valuable inputs to the article and helped in completing the paper.

\section{Conflict of Interest}

The authors have no conflict of interest to declare.

\section{References}

[1] Arias C.R, Jacqueline K. B, Lorrie M. F, Renee M.G, and Mickey E.P. 2002. Yeast Species Associated with Orange Juice: Evaluation of Different Identification Methods. Appl Environ Microbiol, 68(4): 1955-1961.

[2] Chatterjee S, Barnita G, and Rina R R. 2011. Isolation And Characterization of Local Yeast Strains From Waste Fruit Juices, Jaggery, And Dahi Samples. Int. J. Chem. Sci., 9(2): 647-656

[3] Sandeep T, Rajani S, Anjali T, Arjun S, Yuvraj K.C. 2015. Isolation of Yeast from Soil and Different Food Samples and Its Characterization Based on Fermentation. Nepal Journal of Biotechnology, Vol. 3, No. 1: 29-34

[4] Hatcher, W. S., Jr., M. E. Parish, J. L. Weihe, D. F. Splittstoesser, and B. B. Woodward. 2000. Fruit beverages, p. 565-568. In F. P. Downes and K. Ito (ed.). Methods for the Microbial Examination of Food. American Public Health Association, Washington, D.C.

[5] Cherilyn D. G, Jennifer K. S, Patrick L. McD, and Craig A. 2010. Molecular Identification of Veterinary Yeast Isolates by Use of Sequence-Based Analysis of the D1/D2 Region of the Large Ribosomal Subunit. J Clin Microbiol, 48(6): 21402146.

[6] Fell, J. W., T. Boekhout, A. Fonseca, G. Scorzetti, and A. Statzell-Tallman. 2000. Biodiversity and systematics of basidiomycetous yeasts as determined by largesubunit rDNA D1/D2 domain sequence analysis. Int. J. Syst. Evol. Microbiol, 50(Pt. 3):1351-1371.

[7] Sjamsuridzal. W, Oetari A, Gatot F. H, And Sitaresmi. 2006. Pengembangan Database Mikroorganisme Indigenos Indonesia. Makara, Sains, Vol. 10, No. 1, April 2006: 1-5. 
[8] Sjamsuridzal, W. and Oetari, A. (2003). Rapid preparation of fungal and bacterial genomic DNA for PCR. Hayati 10, 122-124.

[9] Maksim Z and Matthias R. 2018. Cell size and morphological properties of yeast Saccharomyces cerevisiae about growth temperature. FEMS Yeast Research, 18:6

[10] Daniel R. 2009. Explaining Lengths and Shapes of Yeast by Scaling Arguments. PLoS One, 4(7): e6205.

[11] Jin SL, Jong HS, Mi NK, Sook IJ, Kyung HP, Duck C, Seung JK, Myung GS, Soon PS, and Dong WR. 2007. Kodamaea ohmeri Isolates from Patients in a University Hospital: Identification, Antifungal Susceptibility, and Pulsed-Field Gel Electrophoresis Analysis. J Clin Microbiol, 45(3): 1005-1010. 